\title{
Fibroblastic Proliferation Present
}

National Cancer Institute

\section{Source}

National Cancer Institute. Fibroblastic Proliferation Present. NCI Thesaurus. Code C96183.

A morphologic finding indicating the presence of fibroblastic hyperplasia in a tissue sample. 